\title{
Electro-Optical Properties of UV Stabilized Sub-Micron Dispersion with Low Liquid Crystal Content
}

\author{
L. Tortora \\ G. Chidichimo \\ University of Calabria, Department of Chemistry, Rende (CS), Italy
}

\section{Kreminska}

\section{A. Golovin}

Kent State University, Liquid Crystal Institute, Kent, Ohio, USA

\section{O. D. Lavrentovich}

Kent State University, Liquid Crystal Institute and Chemical Physics Interdisciplinary Program, Kent, Ohio, USA

We study a nematic dispersion formed in a UV-polymerized matrix with a low content of a liquid crystal component. The dispersions contains sub-micrometer liquid crystal droplets obtained in a two-step process: (a) phase separation during a fast cooling process (b) stabilization of droplets size by photo-polymerization caused by a low power UV exposure. The dispersion is homogeneous, highly transparent and shows little light scattering at wavelength $633 \mathrm{~nm}$. We demonstrate that it can be used as an electrically-controlled birefringent slab with a relatively low driving voltage and a short response time. For example, application of the electric field $6 \mathrm{~V} / \mu \mathrm{m}$ changes phase retardation by $\pi / 4$ with the characteristic response time of several milliseconds.

Keywords: electro-optic effect; optical phase retardation; sub-micron dispersion

\section{INTRODUCTION}

A polymer-dispersed liquid crystal (PDLC) is a composite formed by liquid crystal (usually nematic) droplets dispersed in a polymer matrix $[1,2]$. In the absence of an external field (OFF state), the director

We thank Liou Qiu (Liquid Crystal Institute) for AFM measurements.

Address correspondence to L. Tortora, University of Calabria, Department of Chemistry, 15C Arcauacata di Rende, Rende (CS), 87036, Italy. E-mail: uana.tortora@hotmail.com 
configuration $\mathbf{n}(\mathbf{r})$ within the droplets is determined by the boundary (anchoring) conditions at the liquid crystal/matrix interface and by the bulk elasticity of the liquid crystal [3]. In general, for droplets of a micron size and larger, $\mathbf{n}(\mathbf{r})$ is strongly distorted with topological defects stabilized by surface anchoring [3]. A sufficiently strong electric field is capable to realign the director (along the field if the dielectric anisotropy of the nematic is positive). For the appropriately chosen refractive indices of the nematic and the matrix, the ON state might be adjusted to be transparent and non-scattering, while the OFF state to be strongly light scattering (because of optical inhomogeneities cased by director distortions and liquid crystal-matrix interfaces). The PDLCs find their applications in a variety of shutters, in which one of the states is opaque and another is transparent.

It is clear that light scattering would be diminished in a PDLC with sufficiently small (sub micrometer) droplets; such a material can be used as an electrically-controlled birefringent medium, for example, in phase modulating devices for optical communications [4-8]. The general feature of PDLCs is that as the droplet size decreases, the response time decreases too, but the driving voltage increases [7-9]. One of the problems to solve is thus to reduce the high operating voltage in PDLCs with small droplets. As shown in Ref. [9], reorientation of droplets in a low-viscous low-molecular weight fluid with zero yield stress such as glycerine should occur without any threshold at all, in an arbitrary small field. The disadvantage of using a fluid matrix is that the torque that restores the original (ideally random) orientation of droplets is weak.

In this work, we propose an approach that allows one to achieve both moderate values of the operating voltage and acceptable relaxation (switching-off) times for nematic dispersions with submicronsize droplets, by using a two step preparation process of a mixture with a relatively low liquid crystal content. Such a system is transparent in both OFF and ON states, has a low operating voltage and response time and is capable of significant changes of optical phase retardation.

\section{MATERIALS AND METHODS}

A stabilized dispersion containing submicron-sized liquid crystal droplets was prepared using a mixture of $15 \mathrm{wt} . \%$ of a nematic liquid crystal material TL203 with a positive dielectric anisotropy $\Delta \epsilon=+11$, $\mathrm{n}_{\mathrm{o}}=1.529, \Delta \mathrm{n}=0.2013$ at $\mathrm{T}=20^{\circ} \mathrm{C}$; clearing point $\mathrm{T}_{\mathrm{N}-\mathrm{I}}=77^{\circ} \mathrm{C}$, purchased from Merck, and $85 \mathrm{wt} . \%$ of a blend of UV-curable monomers. The latter was comprised of 39.5 wt.\% of Bisphenol A glycerolate 
diacrylate, $\mathrm{n}=1.5570$ at $\mathrm{T}=20^{\circ} \mathrm{C}$ (Aldrich) and $59.5 \mathrm{wt} . \%$ of CN945B85, $\mathrm{n}=1.4870$ at $\mathrm{T}=20^{\circ} \mathrm{C}$, an aliphatic urethane triacrylate (Cray Valley-Total), doped with $1 \mathrm{wt} . \%$ of UV photoinitiator Irgacure 651 (Ciba-Geigy). The solubility of TL203 in the monomers mentioned above is limited. The mixture was heated to $100^{\circ} \mathrm{C}$ and stirred for good homogenization. The samples were prepared by filling thin cells (thickness was assured by mylar spacers) with indium tin oxide (ITO) electrodes at high temperatures, at which the mixture is not phase separated yet. Phase separation was produced by fast cooling from above the upper critical solution temperature, followed by lowintensity UV irradiation (lamp model UVGL-58, power $3.9 \mu \mathrm{W}$ at $200 \mathrm{~nm}$ ) at low temperature. The irradiation time was set in the range $10 \mathrm{sec}-40 \mathrm{sec}$. For fast cooling, the cells were placed onto the metallic block kept at $\mathrm{T}=-20^{\circ} \mathrm{C}$, or into the Linkam control stage with the liquid nitrogen cooling and temperature controlled in the range from $\mathrm{T}=-10^{\circ} \mathrm{C}$ to $\mathrm{T}=0^{\circ} \mathrm{C}$. Previous studies [10] of a similar mixture of TL202 and Bisphenol A indicate that increasing the rate of cooling and decreasing the concentration of the liquid crystal result in significant reduction of the droplet size. The advantage of our system is that the small phase-separated liquid crystal droplets obtained during the rapid temperature quench are additionally stabilized by UVpolymerization of the matrix.

Light transmittance of the samples was measured at room temperature between two parallel polarizers. The He-Ne laser (Melles Griot) served as a source of light. The wave front generator (WFG500, FLG Electronics) was used to vary the applied voltage at frequency $1 \mathrm{kHz}$. To account for the intensity losses caused by reflection of light at the interfaces (glass/air, glass/ITO, ITO/liquid crystal), we normalized the transmission data by using a cell similar to the tested one with that difference that it contained glycerol instead of the dispersion. Optical transmittance of this cell was used as the reference. The electric-field induced optical phase shift and response times were measured using the Michelson interferometer scheme [11]. Note that, in these measurements, the operating field corresponds to the field magnitude needed to reach $90 \%$ of maximum transmittance of the sample.

\section{EXPERIMENTAL RESULTS}

\section{Polarizing Microscope and AFM Analysis of Morphology}

The structural features of non-polymerized and partially polymerized dispersions are illustrated in Figure 1-3. In absence of polymerization, the temperature-induced phase separation leads to nematic 


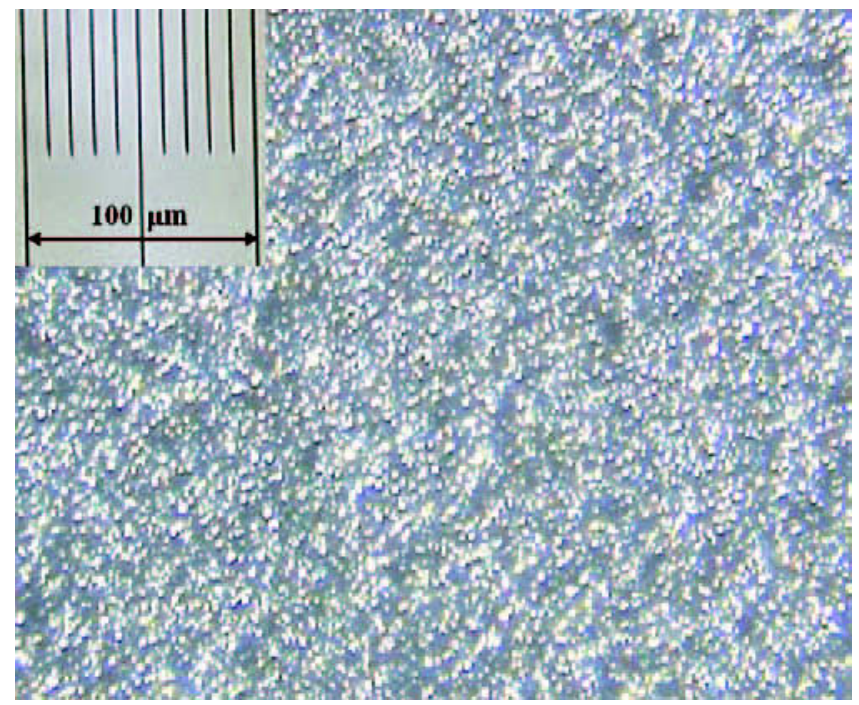

FIGURE 1 Polarizing microscope texture of the non-polymerized dispersion taken at the 4 th day after preparation. The cell thickness was 25 microns.

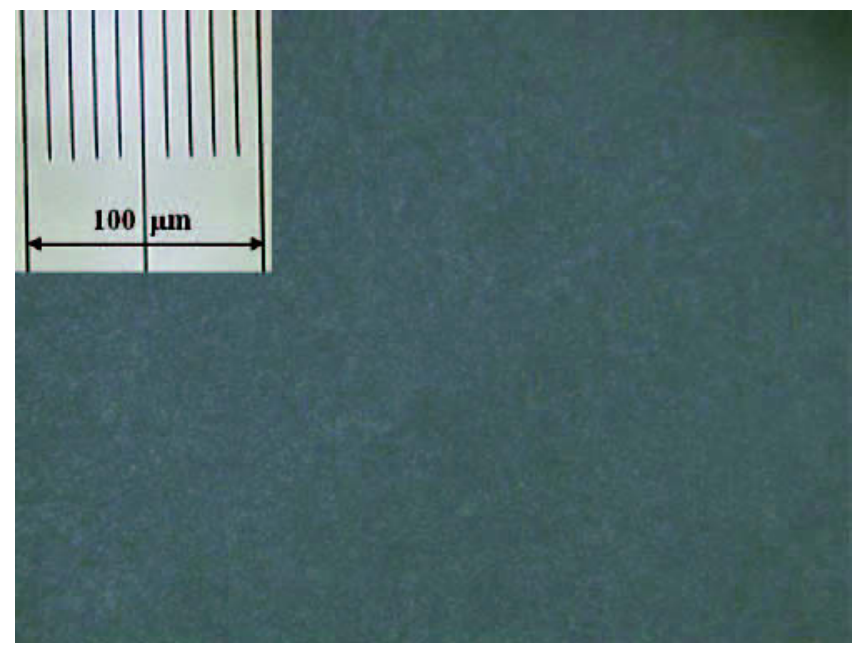

FIGURE 2 Polarizing microscope texture of the stabilized dispersion taken three month after preparation. The dispersion was polymerized by UVexposure for 15 seconds at $(-20)^{\circ} \mathrm{C}$. The cell thickness was 25 microns. 


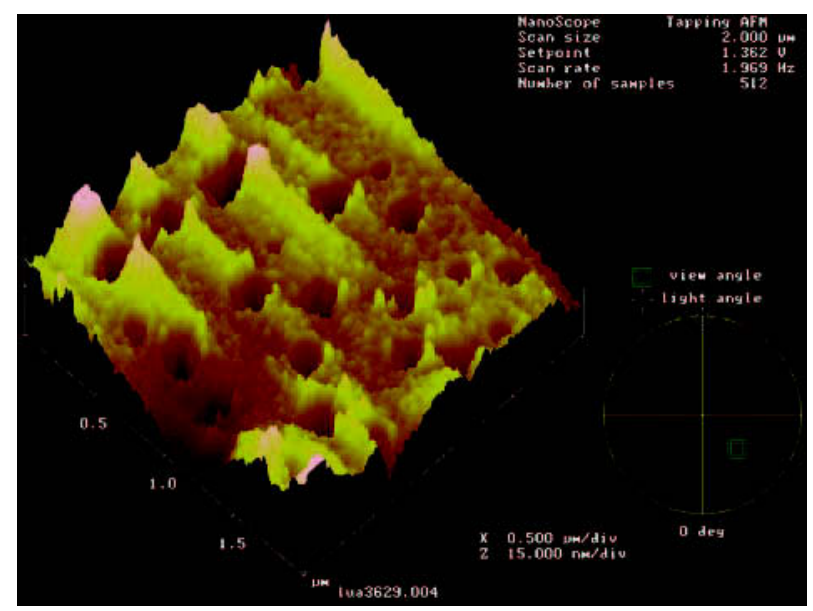

FIGURE 3 AFM image of the polymer part of the dispersion; the matrix was polymerized by $15 \mathrm{sec} \mathrm{UV}$ exposure at $(-20)^{\circ} \mathrm{C}$ and then additionally by 1 hour exposure at room temperature. The maximum size of droplets is about $200 \mathrm{~nm}$.

droplets that are unstable with respect to coalescence and growth. The droplets eventually grow to the micron size (within a few days) and larger (Fig. 1). For the UV-polymerized system, the dispersion is much more stable and the droplets remain submicron, even three month after preparation (Figs 2,3). As evidenced by atomic force microscope (AFM, NanoscopeIIIa, Digital Instruments) observations of the polymerized matrix the typical size of these droplets is of the order of $100 \mathrm{~nm}$ (Fig. 3). The AFM observations were performed for a sample UV-irradiated for 15 seconds at low temperature and then additionally irradiated for 1 hour at room temperature to complete the polymerization.

The content of the liquid crystal in sub-micron emulsions was only 15 wt. $\%$, i.e. smaller than in the previously studied switchable nematic emulsions with $30 \mathrm{wt} \%$ [12]; together with UV-polymerization, it helped to maintain the submicron size of the droplets. The phase separation was not complete even after UV-polymerization, as evidenced by the fact that the dispersion could be transformed into the isotropic state by heating above $55^{\circ} \mathrm{C}-60^{\circ} \mathrm{C}$, which is significantly smaller than the bulk nematic-isotropic transition temperature $\mathrm{T}_{\mathrm{N}-\mathrm{I}}$; the difference should be attributed to the residual quantities of Bisphenol A and CN945B85 inside the nematic droplets. Apparently, the polymerization process was also not complete, as additional 
UV-irradiation resulted in a stiffer matrix and higher operational voltages. Nevertheless, in the absence of direct UV irradiation the samples demonstrated good stability within at least three months after preparation.

\section{Electro-Optical Measurements of Transmittance, Phase Shift and Response Times}

Below we describe the electro-optical properties of a dispersion that was UV-irradiated for 15 seconds while being kept at the stage cooled to $\mathrm{T}=-20^{\circ} \mathrm{C}$. The $28 \mu \mathrm{m}$ thick cell was characterized at room temperature one week after the preparation. First, we determined how light transmission depends on applied voltage (at $1 \mathrm{kHz}$ frequency). The sample was placed between the parallel polarizer and analyzer. The data were normalized so that $100 \%$ corresponded to the transmittance of the cell filled with glycerol. In the OFF state (no electric field) the transmittance of UV-polymerized sample was about $85 \%$, i.e. noticeably higher than the $65 \%$ transmittance of the non-polymerized sample, in which we observed strong scattering. The result indicates that the UV-polymerization helps to stabilize the submicron size of the droplets. When the voltage was increased, the transmission raised to almost $100 \%$.

To determine the switching of optical phase retardation of the sample, we used the $28 \mu \mathrm{m}$ thick cell of a UV-polymerized dispersion in the Michelson interferometer scheme. We used Tektronix TDS 210 digital oscilloscope to register the phase retardation signal detected by photo diode of the interferometer. By increasing the field from 0 to about $6 \mathrm{~V} / \mu \mathrm{m}$, we obtained the phase shift of about $\pi / 4$. Note that the operating field is about 2 times smaller than in the previous studies [5]. To increase the phase retardation, a higher birefringence liquid crystal mixture or a thicker cell gap can be considered. Another possibility is to employ several cells. We also measured the response time of the system, by applying the rectangular voltage pulses of amplitude $170 \mathrm{~V}$ (Fig. 4). The switch-on time $\tau_{\text {on }}$ of structural reorientation when the field was applied was less than $1 \mathrm{~ms}$; the switch-off relaxation time $\tau_{\text {off }}$ was longer, about $5 \mathrm{~ms}$.

Figure 5 demonstrates how the switching time depends on the irradiation time. The switch-on time $\tau_{\text {on }}$ increases to $5 \mathrm{~ms}$ when the UV exposition time is increased to $40 \mathrm{sec}$; in contrast, the switch-off time $\tau_{\text {off }}$ decreases to below $2.5 \mathrm{~ms}$.

The operating voltage can be controlled by changing both the time of UV-irradiation and temperature at which the sample was irradiated (Fig. 6). For samples polymerized at $\mathrm{T}=-10^{\circ} \mathrm{C}$ during 


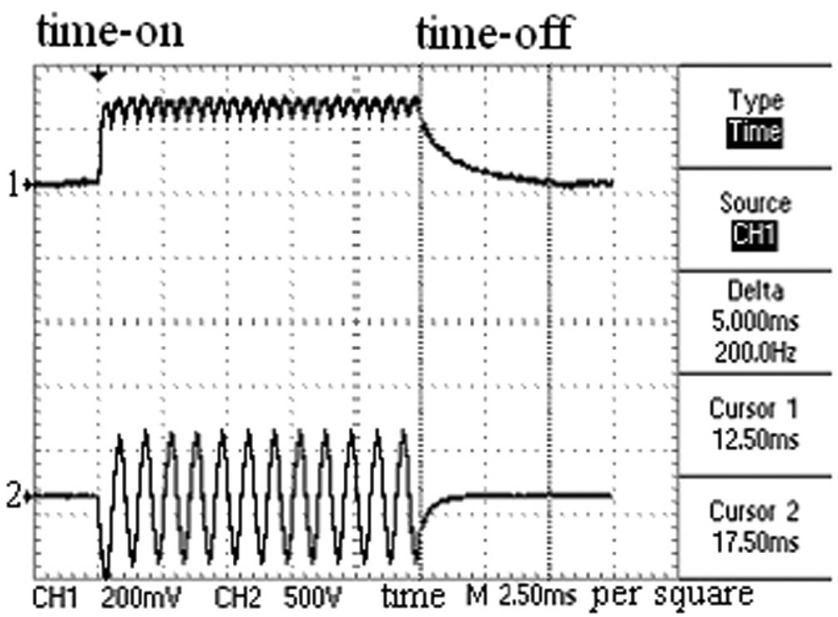

FIGURE 4 The oscilloscopic picture of the signal from a photodetector in the Michelson interferometer (top curve 1) versus the applied voltage (bottom curve 2). The voltage amplitude changes from 0 to $170 \mathrm{~V}$ abruptly at the carrier frequency of $1 \mathrm{kHz}$. The cell thickness is $28 \mu \mathrm{m}$; the dispersion was $\mathrm{UV}$-polymerized for $15 \mathrm{sec}$ at $(-20)^{\circ} \mathrm{C}$.

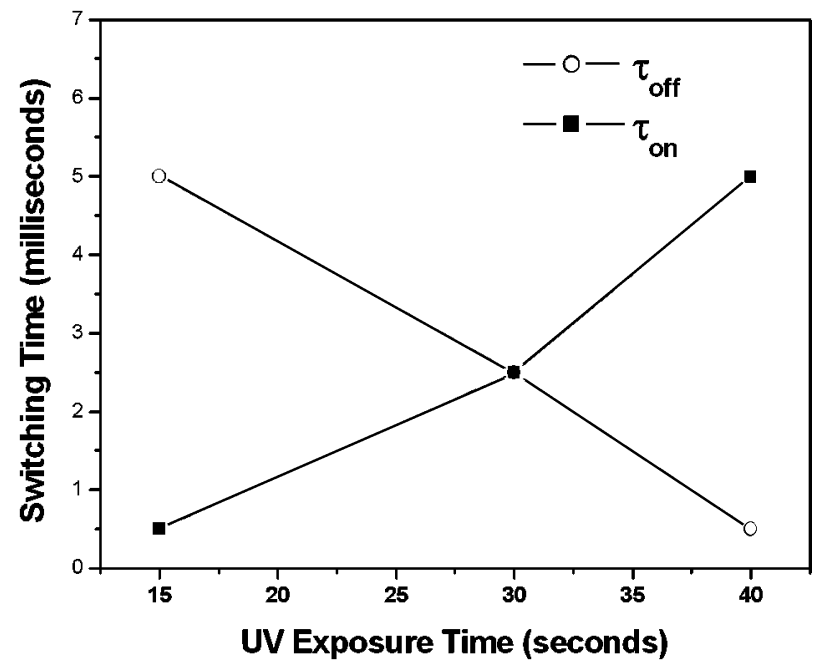

FIGURE 5 The effect of UV-exposure time on switching times of dispersions irradiated at $(-20)^{\circ} \mathrm{C}$. 


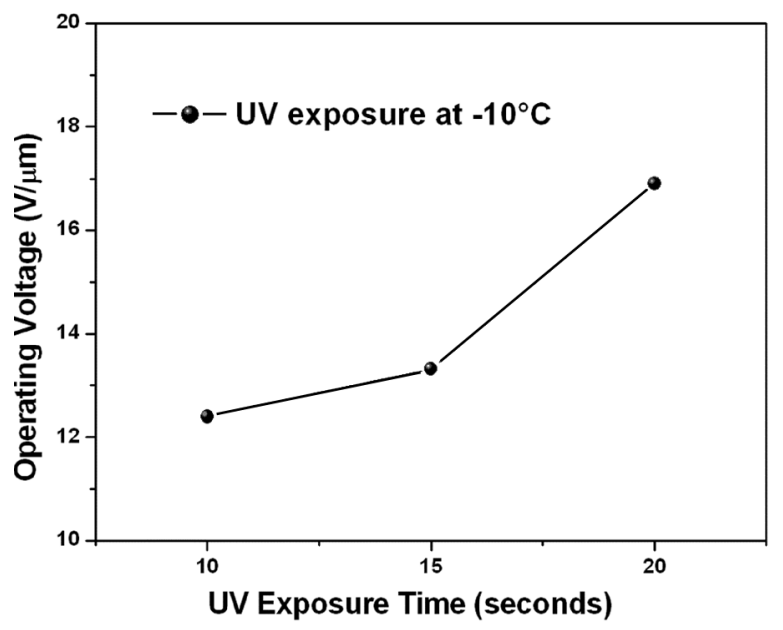

(a)

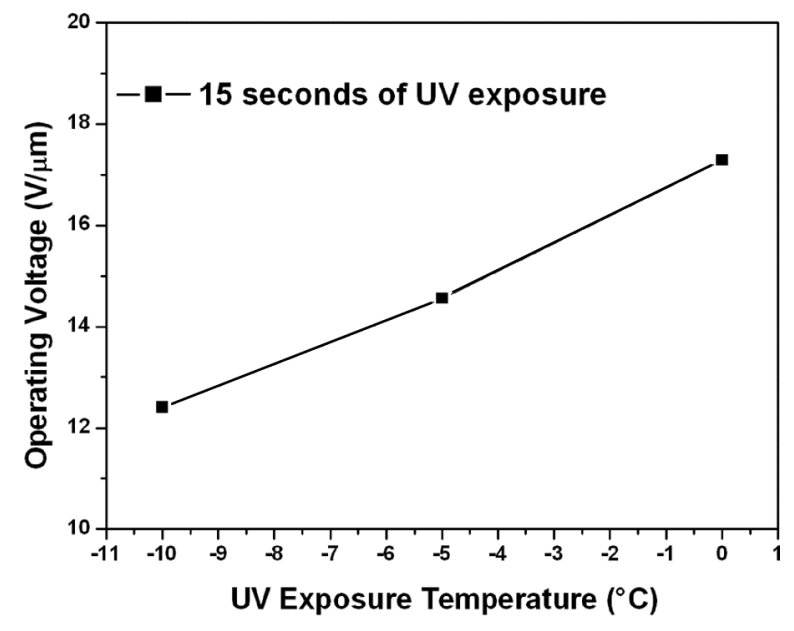

(b)

FIGURE 6 The effect of (a) duration of UV-irradiation and (b) temperature of irradiation on the operating voltage of the dispersion.

10 seconds the operating field is $12 \mathrm{~V} / \mu \mathrm{m}$. It increases with the increase of the irradiation time and temperature. Optical transmittance of these samples varies from $85 \%$ (OFF state) to $100 \%(\mathrm{ON}$ state) with respect to the reference glycerol cell, and it does not change significantly with the polymerization conditions (Fig. 7). 


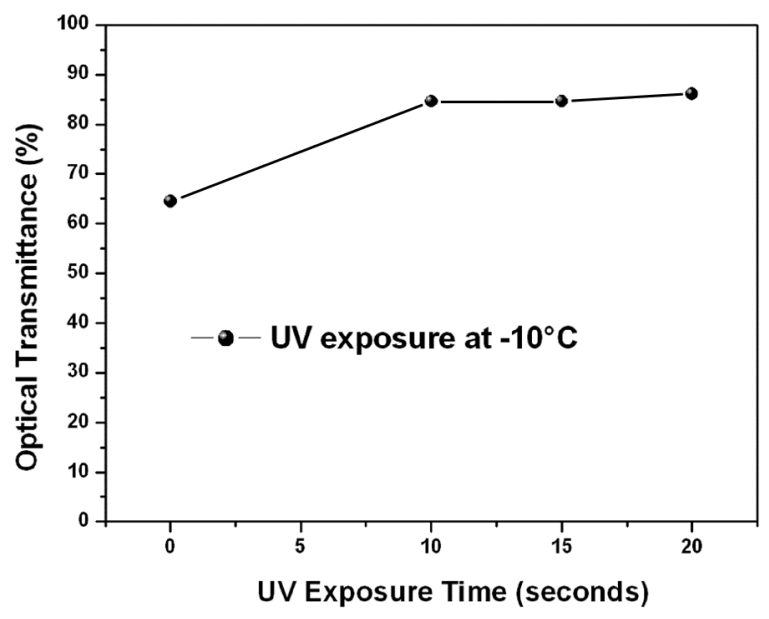

(a)

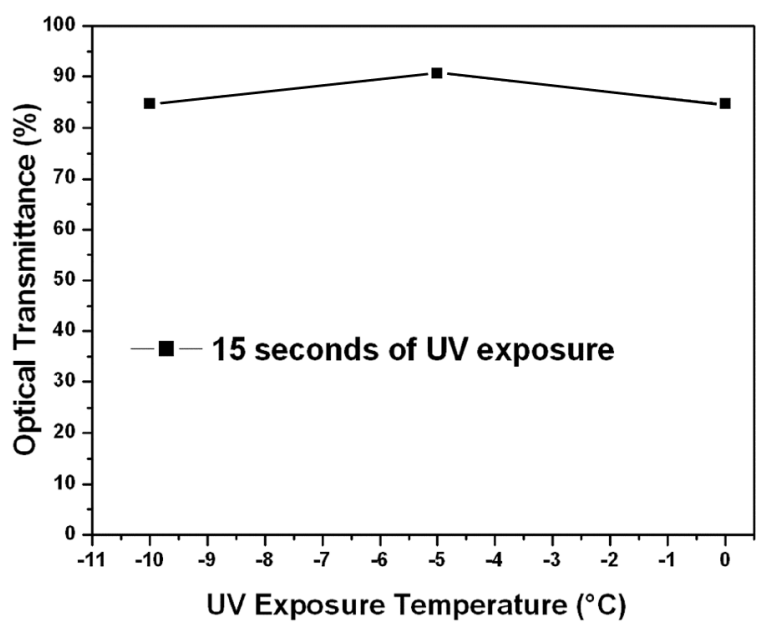

(b)

FIGURE 7 The effect of (a) duration of UV exposure and (b) temperature of irradiation on light transmittance of the cell (thickness $25 \mu \mathrm{m}$ ).

\section{DISCUSSION AND CONCLUSION}

The operating voltage, relaxation times, and other aspects of droplets reorientation in nematic emulsions are determined by a number of factors, including the elastic and surface anchoring properties of the liquid crystals and the nature of the matrix, in particular, its 
viscoelastic properties. From the point of view of surface anchoring, our system corresponds to the case of tangential anchoring [9], as the director tends to be parallel to the interface. It can be easily verified by observing the textures of large $(10-30 \mu \mathrm{m})$ droplets that demonstrate a characteristic bipolar director configuration with two point defects-boojums at the poles of the droplets [1,3]; the director lines are tangential to the surface. Note, however, that for our sub-micron droplets we expect that instead of the well-pronounced bipolar configuration, the director becomes practically uniform within each droplet (but can change from droplet to droplet in absence of the external field). Such a behaviour is expected for droplets with radii $R<K / W$, where $K$ is the typical Frank constant and $W$ is the typical (polar) anchoring coefficient: the director tends to be uniform at the expense of the violated surface orientation, as the associated surface energy $\propto W R^{2}$ is much smaller than the energy of the elastic distortions $\propto K R$ needed to satisfy the boundary conditions [3]. Typically, $K / W$ is of the order of $1 \mu \mathrm{m}$ [3]. Once such a droplet with a practically uniform director is aligned, there is no restoring torque to deviate the director from this orientation when the field is switched off (except for random factors such as Brownian effects); the corresponding reorientation times are very large, as already determined for the similar non-polymerized emulsions in the previous studies [10,12,13].

In fluid matrices such as glycerine, field-induced realignment of bipolar and similar droplets involves two processes: reorientation of the droplet as the whole which is accompanied by the flow of the matrix around the droplet and director reorientation within the droplet [9]. In the fluid matrices, the droplet reorientation can start and be completed at negligibly small fields through the rotation of the whole droplet, the drawback is that the restoring torque (after the field is switched off) is small, as discussed above. In rigid polymer or glassy matrices, the reorientation dynamics is defined by the director realignment and the restoring torque is significant, as it can be caused by the irregularities of the droplets shape or by trapping of the boojums at the droplets surface [9]. In this work, the goal was to achieve an intermediate situation, by making the matrix sufficiently rigid to cause director and droplet relaxation back to the random (or partially random) director orientation in the $\mathrm{ON}$ state and yet at the same time sufficiently fluid-like to keep the operating voltage low. We prepared the nematic dispersion by a fast temperature quench of a mixture with a low content of the liquid crystal and used a subsequent UVpolymerization at low temperature to prevent the droplet growth. Although the chosen system is certainly not optimized for the best possible performance, it does demonstrate the desirable features. 
First of all, we demonstrated that the dispersion contains sub-micron size droplets and does not show noticeable morphology changes within at least three month. As the droplets are of a submicron size, light scattering is mitigated and the cells of thickness $28 \mu \mathrm{m}$ demonstrate transparency at the level of $85 \%$ or better. By applying the electric field, one reorients the director and thus changes the effective optical retardation of the cell. We demonstrated that a phase shift of $\pi / 4$ (at the wavelength $633 \mathrm{~nm}$ ) is achievable when the $28 \mu \mathrm{m}$-thick cell is operated by the filed $6 \mathrm{~V} / \mu \mathrm{m}$. Therefore, such a stabilized dispersion can be used in fast phase modulating devices. One of the most urgent tasks is to verify whether the system preserves its properties over a period of time significantly longer than three months. If needed, further stabilization of the system can be achieved in a variety of ways, for example, by adding surfactants or using polymer gels. This work is in progress.

\section{REFERENCES}

[1] Drzaic, P. D. (1995). Liquid Crystal Dispersions, World Scientific: Singapore.

[2] Doane, J. W., Golemme, A., Wu, B. G., \& Zumer, S. (1986). Appl. Phys. Lett., 48, 269.

[3] Kleman, M. \& Lavrentovich, O. D. (2003). Soft Matter Physics: An Introduction, Springer: New York.

[4] Matsumoto, S., Sugiyama, Y., Sakata, S., \& Hayashi, T. (1999). J. of Intelligent Material Systems and Structures, 10, 489.

[5] Lucchetta, D. E., Manni, A., Karapinar, R., Gobbi, L., \& Simoni, F. Mol. Cryst. Liq. Cryst., 375, 397.

[6] Ren, H. \& Wu, S. T. (2002). Appl. Phys. Lett., 81, 3537.

[7] Sutherland, R. L., Tondiglia, V. P., \& Natarajan, L. V. (1994). Appl. Phys. Lett., 64, 1074.

[8] Bunning, T. J., Natarajan, L. V., Tondiglia, V. P., \& Sutherland R. L. (2000). Ann. Rev. Mater. Sci., 30, 83.

[9] Koval'chuk, A. V., Kurik, M. V., Lavrentovich, O. D., \& Sergan, V. V. (1988). Zh. Exp. Teor. Fiz., 94, 350; [(1988). Sov. Phys. JETP., 67, 1065].

[10] De Filpo, G., Lanzo, J., Nicoletta, F. P., \& Chidichimo, G. (1998). J. Appl. Phys., $84,3581$.

[11] Born, M. \& Wolf, E. (1970). Principles of Optics, Pergamon Press: Great Britain.

[12] Lanzo, J., Nicoletta, F. P., De Filpo, G., \& Chidichimo, G. (2000). Liquid Cryst., $27,1029$.

[13] De Filpo, G., Lanzo, J., Nicoletta, F. P., \& Chidichimo, G. (1999). J. Appl. Phys., $85,2894$. 